\title{
QUANTUM CONTROL OF MOTIONAL STATES OF NEUTRAL ATOMS: EXPLOITING THE EXTERNAL DEGREES OF FREEDOM
}

\author{
N.P. Bigelow, W. Chalupczak, R. Ejnisman, H. Pu, P. Rudy \\ AND J. SHAFFER
}

Department of Physics and Astronomy and the Laboratory for Laser Energetics The University of Rochester

Rochester, NY 14627, USA

\begin{abstract}
As research in quantum optics has advanced, so too has our ability to precisely tailor the quantum state of a system. Indeed, techniques for quantum state preparation have become sufficiently advanced that an entire subfield has appeared which has been given the name "quantum control". Parallel to these advances have been other striking developments in quantum optics, in particular, laser cooling and trapping of neutral atoms. In this paper we describe some of the recent advancements in laser cooling, particularly in our laboratories, and point out that laser cooling and trapping is also realizing an important form of quantum control. In laser cooling, instead of exercising control over the internal quantum state of an atom or molecule or a laser field, we are instead controlling a complementary set of degrees of freedom: those of the external coordinates of the atom.
\end{abstract}

PACS numbers: 03.75.Fi, 32.80.Pj, 42.50.Dv

\section{One atom interacting with many photons: laser cooling and trapping}

\subsection{Manipulating the atomic velocity: laser cooling basics}

The idea that atoms could be slowed and cooled by their interaction with light was first proposed in 1975 [1] and demonstrated shortly thereafter on trapped ions in 1978 [2]. It was not, however, until 1981 that a group at the Institute of Spectroscopy in Moscow demonstrated the application of laser cooling to the slowing of a neutral atomic beam [3]. Soon afterwards, a group at the National Institute of Standards and Technology (NIST) reported the cooling of an atomic beam to temperatures of less than $100 \mathrm{mK}$ [4]. This accomplishment opened the door to the realization of the three-dimensional cooling of a vapor of neutral sodium atoms to a few hundred microkelvin by a group at Bell Laboratories in 1985 and 
the subsequent cooling and trapping of an atomic vapor using the now ubiquitous magneto-optical trap (MOT) in 1987 by the same group [5].

Formally, we can derive expressions for the light pressure force on an atom interacting with a laser field by starting from the Lorentz force. In practice, a detailed microscopic understanding of the light pressure force which is valid under all conditions is complex and in some cases incomplete. However, by restricting the situation to moderate atomic velocities and moderate laser field intensities, a convenient decomposition of the force can be made [6].

All of the forces involved in the optical cooling and trapping of atoms hinge on the exchange of energy and momentum between the atom, the laser field, and the vacuum; an exchange which occurs through photon absorption and emission. In this paper we consider two classes of interaction. One of these concerns processes in which the atom absorbs a photon and then reemits it spontaneously some characteristic time $\tau_{\mathrm{sp}} \equiv 1 / \Gamma$ later. The other class of processes are those which involve stimulated emission. In many situations we can associate each of these two types of processes with distinct force mechanisms: the spontaneous and the dipole forces.

The first realization of an optical trap was based on the force which emphasizes stimulated emission of photons: the dipole force. In a semi-classical treatment, the dipole force arises from the interaction between the quadrature component of the dipole moment induced in the atom by the laser field and the gradient of the field itself. This interaction produces a shift in the atomic energy levels via the AC Stark shift. If there is a spatial gradient in this shift, then the atom experiences a net force. In a more quantum mechanical treatment, the dipole force is understood in terms of the momentum transfer to the atom which occurs as the atom mediates the transfer of photons from one mode of the applied laser field to another. Because this transfer occurs at constant photon energy the dipole force is a conservative force. Indeed, for a two-level atom, the dipole force can be conveniently expressed in terms of a potential $U(x)=-\hbar \Delta \ln [1+p(x)]$ where $p(x)=\left[I(x) / I_{\text {sat }}\right] \Gamma^{2} /\left(4 \Delta^{2}+\Gamma^{2}\right)$. Here $I_{\text {sat }}$ is the two-level transition saturation intensity and $\Delta=\omega_{0}-\omega_{\text {laser }}$ is the laser field detuning from the atomic resonance. For moderate field intensities and/or large detunings $U(\boldsymbol{x}) \propto I(\boldsymbol{x})$ and hence $\boldsymbol{F} \propto \nabla I(x)$.

Cooling of an atom arises from the dissipation of kinetic energy and hence forces which can cool an atomic vapor are intrinsically nonconservative. In laser cooling, this is generally achieved by absorbing a photon of one wavelength and emitting a photon of a different wavelength, the difference in photon energy ( $\Delta E=$ $\left.h c / \lambda_{\text {emit }}-h c / \lambda_{\text {absorb }}\right)$ being provided by the change in atomic kinetic energy. The simplest such cooling processes are referred to as the dissipative or spontaneous force and with a particular laser field configuration which has been named "optical molasses".

Consider a two-level atom, initially in its ground state $|g\rangle$ with center-of-mass momentum $\boldsymbol{P}$ interacting with an infinite plane traveling wave characterized by a wave vector $k$. When the atom absorbs a single photon from this wave the atomic momentum changes by $\hbar k$ and the atom emerges in the excited state $|e\rangle$. Simply put, the atom receives a momentum kick along $k$. Some characteristic time later 
$\left(\tau_{\mathrm{sp}}\right)$ the atom spontaneously decays back to the ground state $|g\rangle$, reemitting a photon with wave vector $k^{\prime}$, receiving another momentum kick: $\hbar k+|g, \boldsymbol{P}\rangle \rightarrow$ $|e, \boldsymbol{P}+\hbar k\rangle \rightarrow \hbar k^{\prime}+\left|g, \boldsymbol{P}+\hbar\left(k-k^{\prime}\right)\right\rangle$. If we consider the average over $n$ cycles then as $n \rightarrow \infty$ the state of the atom approaches $|g, P+n \hbar k\rangle$ : a net momentum $n \hbar k \cdot 1_{1}$ as been transferred to the atom over a time scale of approximately $n \tau_{\text {sp }}$ (Fig. 1). To estimate the size of the resulting force, consider the case of a sodium atom $\left(M_{\mathrm{Na}}=23 \mathrm{amu}\right)$ absorbing light which is nearly resonant with the $D 2$ line $(589 \mathrm{~nm})$. The change in momentum, $\hbar k$, in one absorption/spontaneous emission cycle $\left(\tau_{\mathrm{sp}}=16 \mathrm{~ns}\right)$ gives an acceleration, $a=\hbar k \Gamma / 2 M_{\mathrm{Na}}$ of the order of $10^{6} \mathrm{~m} / \mathrm{s}^{2}$ $\left(10^{5} g !\right)$.

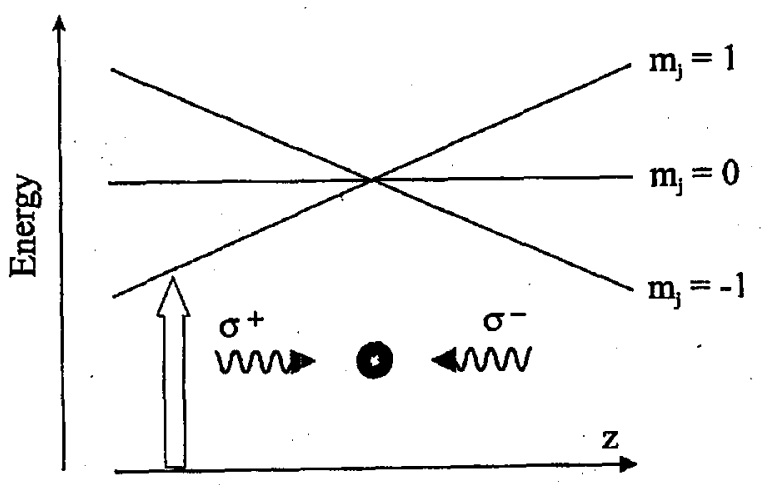

Fig. 1. The operation of a magneto-optical trap for a $J=0 \rightarrow J=1$ transition: As the atom propagates to either the left or the right it is Zeeman shifted into resonance with the laser beam that it is counterpropagating with respect to its displacement and it is "pushed" by the dissipative force back towards the magnetic field zero. This interaction provides the restoring or trapping force. If the laser is also detuned below the atomic resonance, the cooling effect of optical molasses (see Fig. 2) will simultaneously cool the atom.

If the light field is an optical standing wave formed from two counter-propagating infinite traveling waves, and the field frequency is tuned below (to the red of) the two-level transition frequency (i.e. $\Delta<0$ ), then the spontaneous force can generate a velocity dependent force which causes a damping of the atomic velocity. The velocity damping - the cooling - arises from the fact that an atom with a finite velocity will be Doppler shifted into resonance with the traveling wave component of the standing wave which is directed opposite to the atomic motion. The atom will therefore absorb more photons per unit time from the counter propagating wave than from the copropagating wave and will experience a net force which opposes its motion. The result is a velocity dependent force which causes the atom to decelerate regardless of the direction of atomic motion. This standing wave configuration is referred to as optical molasses.

The energy flow in optical molasses can be understood semi-classically by noting that when the atom re-emits a photon spontaneously, this photon is emitted 
at the atom's rest frame resonance frequency $\omega_{0}$ and this photon is higher in energy than that of the absorbed photon by an amount equal to the Doppler shift $\hbar \Delta=\hbar \boldsymbol{k} \cdot \boldsymbol{v}$. To conserve energy, the kinetic energy of the atom decreases and the atom is cooled. In a more quantum mechanical picture, the cooling can be understood as the irreversible scattering of photons out of the laser field and into the empty electromagnetic field modes of the vacuum.

The steady-state temperature for a gas of atoms in equilibrium with an optical molasses is determined by the balance between the Doppler cooling and the spontaneous or diffusive heating associated with the individual random velocity recoil kicks experienced during spontaneous emission. In the limit where the atom is modeled as a simple two-level system it is predicted [6] that the vapor will equilibrate at the so-called Doppler temperature $T_{\text {Doppler }}=\hbar \Gamma / 2 k_{\mathrm{B}}$ which, for sodium, is $240 \mu \mathrm{K}$. Clearly then optical molasses enables us to modify one important external coordinate: the atomic center-of-mass velocity.

To the surprise of many researchers, it was discovered in 1988 by the group at NIST that the atoms in optical molasses could be cooled to well below the Doppler temperature. Indeed, more recently, temperatures below $2 \mu \mathrm{K}$ (barely a few photon recoil kicks of average momentum!) have been achieved for $\mathrm{Cs}$ atoms using optical molasses [6]. The microscopic explanation for this remarkable super-cooling power of optical molasses involves two facts. First, the atoms are not two-level systems; they have a complex magnetic substructure that cannot be neglected. Second, the light field of optical molasses usually involves complex spatially varying intensities and polarizations which also cannot be ignored. These two facts give rise to a new time scale: the time scale for motion of population amongst these internal states via an effect referred to as optical pumping [7]. This microscopic mechanism was described by a beautiful model introduced by the Paris group and is now referred to generally as "polarization gradient" cooling.

\subsection{Manipulating the atomic position: trap basics and the MOT}

Although optical molasses can be used to modify the velocity distribution of a vapor of atoms, it cannot necessarily be used to control their position and hence to confine them in space. To accomplish this task, we need to create a restoring force centered about some position in space, or, in other words, we need to create a trap. More than any other single device, the magneto-optical trap is the hallmark of laser cooling and trapping.

In the MOT, the trapping force is also produced using the scattering force. This is achieved by introducing a spatially varying Zeeman shift which acts much like the Doppler shift does, except in position space rather than velocity space [5]. Figure 1 illustrates a simple one-dimensional model of how the MOT functions for the case of a $J=0 \rightarrow J=1$ atomic transition. A quadrupolar field, produced by a set of magnetic field coils, creates a linear magnetic field gradient which increases from a zero located at the center of the trap. As in optical molasses, the laser light is detuned to the red of the atomic resonance but now we also require that each of the counterpropagating fields has opposite circular polarization: $\sigma^{+}$ traveling in the $z+$ direction and $\sigma^{-}$traveling in the $z$-direction. Now, if the atom is displaced from the field zero (i.e. the trap center) the upper level $(J=1)$ 
manifold will be Zeeman split into its magnetic sublevels $m_{J}$ as shown in Fig. 1 . Because of the careful choice of field polarizations, the sub-level which is shifted lower in energy will also be shifted into resonance with the counterpropagating laser beam and be pushed back towards trap center. The end result is a restoring force towards the magnetic field zero and hence the formation of a trap. Because the laser fields are tuned to the red of resonance $\Delta<0$ the atoms are also cooled by the molasses and by polarization gradient effects such that the MOT both cools and traps the atoms. The temperatures in the real MOT range from a few $T_{\text {recoil }}$ (where $T_{\text {recoil }}=\hbar^{2} k^{2} / 2 k_{\mathrm{B}} M$ ) to a few $T_{\text {Doppler }}$ depending on the intensities and detunings of the trapping laser beams. The record densities for the MOT are $>10^{11}$ atoms $/ \mathrm{cm}^{3}$ and the total number of atoms held in a MOT can exceed $10^{9}$ atoms [8].

\section{Many individual atoms + many photons: optical lattices and parametric excitation of motional states.}

\subsection{The optical lattice}

When multiple laser beams overlap in space they can interfere. An atom placed in this interference pattern will experience the spatial variations of the electromagnetic field. The result is that there are forces acting upon the atoms on the scale of an optical wavelength. For a two-level atom, these forces will be exactly the dipole force described earlier. To describe the evolution of an atom moving in the interference pattern, we can think in terms of a potential surface $U(x) \propto I(x)$ which directly reflects the one, two or three-dimensional periodicity of the optical interference pattern. So far we have stressed the way in which this potential surface contributes to cooling of atoms whose energy is large enough that they can climb the hills of $U(x)$. However, as the atoms evolve on this surface they can reach a low enough total energy that they can become localized in the periodic minima for long periods of time. When this occurs, a highly organized array of atoms is created which is referred to as an optical lattice. Optical lattices have played an important role in a variety of experiments. In one class of experiments, optical lattices have been used as a model. of the familiar solid-state crystal and the correspondence between these systems has been extensively studied. In the second class of experiments, optical lattices have been used as a tool for controlling the motional state of the individual atomic de Broglie waves.

In many ways, the optical lattice is a novel model of the solid-state where the atoms play the role of a gas of electrons interacting with a periodic potential. Many exciting optical lattice experiments have been carried out which probe this analogy. Unlike a true solid, however, the lattice potential in an optical lattice is externally imposed. As a result, it is as if the periodic potential exists whether the lattice is fully occupied or not, maintaining its perfect order. For this reason optical lattices can and are used to explore another very interesting limit inaccessible in the true solid-state: the highly dilute limit where the atoms behave as isolated particles arranged randomly on the lattice grid. In this way the optical lattice still displays perfect long-range order even though most of the lattice sites may be empty! This last fact has recently been demonstrated in an elegant set of experiments in which 
a probe laser beam was Bragg diffracted from an optical lattice [9] despite the fact that less than one in ten of the lattice sites was occupied by an atom.

The first experiments which showed that atoms could be localized in an 3D optical lattice were based on spectroscopy of light scattered by atoms in an optical molasses lattice [10]. In these experiments the NIST group used homodyne spectroscopy to show that a narrow peak less than $50 \mathrm{kHz}$ wide was present in the spectrum even though the natural line width of the atoms was much much larger $(\approx 10 \mathrm{MHz})$. This peak was attributed to the motional narrowing predicted by Dicke [11] for a radiator confined within a potential well. Soon thereafter, these experiments were improved and distinct side bands were observed on this peak. The presence of these side bands proved that not only were the atoms confined in the lattice wells but that the bound states in the wells were clearly and resolvably quantized (see Fig. 2). Since that time a variety of lattice symmetries have been investigated, representing a "crystallography" of the optical lattice and well known properties such as magnetic susceptibility of the lattice have been measured optically [12].

Some of the important properties of a gas of electrons in a solid include the ideas of Bloch waves, energy band curvature and effective mass - all properties which relate to the presence of extended states. In a recent set of experiments

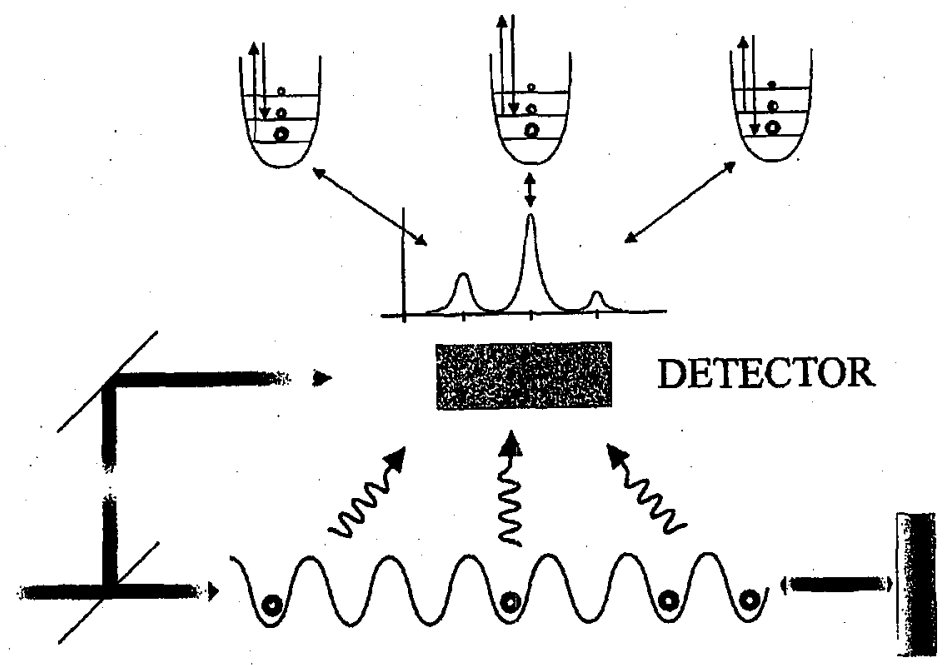

Fig. 2. In an optical lattice atoms are trapped in the interference patterns produced by overlapping multiple laser beams. The motional state of the atom localized in each lattice potential well is shown to be quantized using homodyne spectroscopy. In this experiment, a portion of the lattice light was mixed with the scattered light and the spectrum of the beat note was studied. The beat signal showed three well resolved peaks corresponding to spontaneous Raman transitions between the bound states of the lattice. The different amplitudes of the two side bands of the central peak reflects the fact that the population of the lower bound states is larger than the upper bound states. This ratio can be used to measure the temperature distribution of the localized atoms. 
these effects have also been observed in optical lattices [13]. In what follows, we briefly describe the beautiful experiment carried out by Paris group in which Bloch oscillations were observed [14].

Bloch oscillations are a purely quantum mechanical effect predicted by Felix Bloch decades ago. The prediction was that an electron experiencing a constant force, and hence constant acceleration, will display oscillations in its momentum. These oscillations occur because as the electron accelerates up to the edge of the Brillouin zone its quasi-momentum will effectively fold back around the zone causing the momentum to reverse. This reversal takes place every time the particle accelerates to the zone boundary. To mimic a constant acceleration, the Paris group accelerated the optical lattice and simultaneously measured, as a function of time, the momentum distribution of the atoms in the lattice. Clear oscillations were observed in this momentum at exactly the Bloch period. Moreover, a detailed analysis of the data clearly revealed the signature of extended states through effects such as the curvature of the energy bands of the optical lattice. It is interesting to note that although Bloch oscillations are a fundamental idea in solid-state theory, they are essentially impossible to observe in natural crystals because of electron scattering from lattice defects. In the optical lattice, the periodicity of the lattice potential is nearly perfect, defect scattering is essentially absent, and the Bloch oscillations gracefully emerge. A series of closely related experiments were carried out by a group in Texas who have also used optical lattices as a system for studying the physics of Wannier-Stark ladders, tunneling and quantum chaos [15].

\subsection{Parametric excitation of motional wave packets in an optical lattice}

Groups in Japan, at NIST, in Munich and our group at Rochester [16], have also been using optical lattices to explore another interesting problem: the quantum control of center-of-mass wave packet motion in the optical lattice. In these experiments we have taken advantage of the fact that the potential wells which bind the atoms in the lattice can be manipulated rapidly and with great precision. For example, by changing the light field parameters of the lattice beams as a function of time, the atoms in the lattice can be excited into novel motional states and the evolution of these states can be carefully studied. Simply put, by dynamically controlling the spring constants and origins of the lattice wells, the atomic wave packets in the lattice can be tailored and caused to vibrate and move in a highly controlled manner.

In our experiments we achieved this effect by modulating the laser field intensity and detuning as a function of time. If we view the atom as a nearly harmonic oscillator, our technique corresponds to the familiar parametric excitation of that oscillator. In particular, consider a single atom bound in a single well. If the spring constant of the well is suddenly decreased, then a wave packet originally stationary in the center of the original well will begin to execute a breathing motion. In a quantum picture this is because when the initial wave packet state is projected onto the bound states of the final well the resulting superposition state evolves in time in a manner that produces the breathing motion. In our experiments we probe the wave packet evolution by time resolved spectroscopy of the fluorescence from the lattice atoms. This technique relies on the fact that the lattice potential 


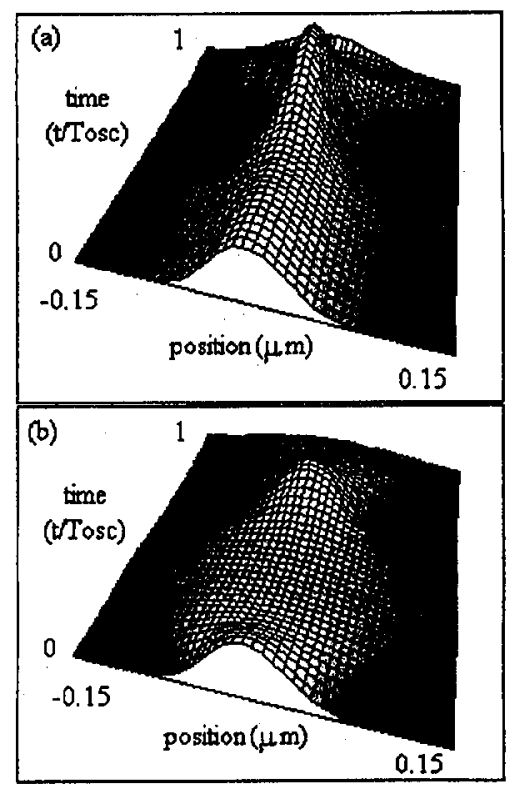

Fig. 3. The spring constant of the lattice potentials are modulated in time to induce coherent motion of the atomic wave packets bound in the lattice potentials. In these experiments the motion is detected through time resolved fluorescence measurements. This technique takes advantage of the fact that the lattice potential $U(x) \propto I(x)$. The result is that as the atoms vibrate, they explore different laser intensities, causing their fuorescence to vary in time. From this data we can reconstruct the wave packet motion and compare the desired wave packet motion as calculated analytically. This experiment clearly demonstrates the ability to use optical lattices to execute a novel form of quantum control (from P. Rudy et al., Ref. [16]).

scales as $U(x) \propto I(x)$. Since the fluorescence rate depends on $I(x)$, and $x=x(t)$, as the packet moves the fluorescence is a measure of the wave packet distribution in the well. In Fig. 3 we show the time evolution of an atomic wave packet in a driven optical lattice as reconstructed from data taken when $U(x)$ was varied linearly in time. We also show a reconstruction of the predicted and measured wave packet for this experiment. The shape and evolution of this wave packet are in good agreement with our theoretical predictions for this $U(x, t)$ demonstrating our ability to use a pre-tailored $U(x, t)$ to generate a desired wave packet evolution.

In our optical lattice wave packet experiments, the oscillations were observed to decay after several oscillation cycles. Typical decay data for a given set of initial conditions (laser intensity, detuning, sample temperature, etc.) is shown in Fig. 4. A fit to this data yields a decay time of $\approx 15 \mu \mathrm{s}$. The origin of the wave packet damping for our experiments can be understood in terms of an interplay of anharmonicity induced dephasing and irreversible damping due to spontaneous emission. To estimate the dephasing rate due to anharmonicity in the lattice potential, we consider the first order correction to the sinusoidal potential and find 


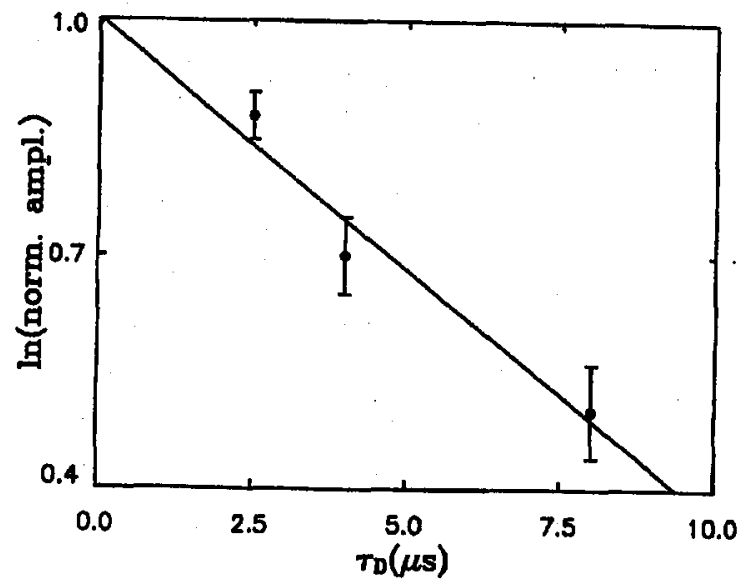

Fig. 4. Decay rates of parametrically excited optical lattice wave packets. The best-fit slope gives a decay constant of $15 \mu \mathrm{s}$.

$T_{\text {an }} \propto 1 /\left(j^{2}+j+1 / 2\right)$, where $j$ is the mean level index for the levels used to form the wave packet. In our experiments $j \approx 1$ and we find $\tau_{\mathrm{an}} \approx 10 \mu \mathrm{s}$, in reasonable agreement with the observed decay time. If, on the other hand, we estimate the decay time expected from spontaneous emission, treating spontaneous emission as a random walk in frequency space, we find $\tau_{\text {sp-decay }} \propto \omega_{\text {osc }} / \omega_{\text {recoil }} R$, where $\omega_{\text {osc }}$ is the harmonic frequency for wave packet oscillation in the well, $\omega_{\text {recoil }}$ is the single photon recoil shift and $R$ is the spontaneous emission rate. For our experimental conditions, $\tau_{\mathrm{sp}-\text { decay }} \approx 10 \mu \mathrm{s}$ also! In other words, in our experiment we cannot discriminate between these two decay processes. One very important conclusion is, given that spontaneous dephasing is present, there is little hope of observing quantum revivals [17] in our current experiment - irreversible decay will mask such quantum effects. If, however, we repeat our experiments for larger detunings (which reduces the spontaneous emission rates) such revivals may be observable.

Quantum revivals can be expected to occur in the optical lattice due to the fact that the lattice wells are not perfectly harmonic. For the optical lattice, the observation of revivals would provide dramatic proof of the quantum nature of the system. To investigate this possibility we have carried out a numerical study of wave packet evolution in the lattice [18]. In particular, we have solved Schrödinger's equation numerically and modeled the time evolution of a wave packet created at $t=0$ from a superposition of the bound lattice states. For simplicity, we have neglected band structure effects. For deeper lattice wells, and for wave packets involving mostly the low lying states, band structure effects should be unimportant. However, we note that for shallow potentials, well-to-well tunneling may become important and band curvature may not be negligible. In Fig. 5 we show the autocorrelation function between the initial wave packet and the wave packet at a time $t$ after a sudden change of the lattice well. Here $C(t)=\int \psi^{*}(x, 0) \psi(x, t) \mathrm{d} x$. Not only is there a clear revival seen, but there are well resolved fractional revivals. In the inset, we show the norm of the initial wave 


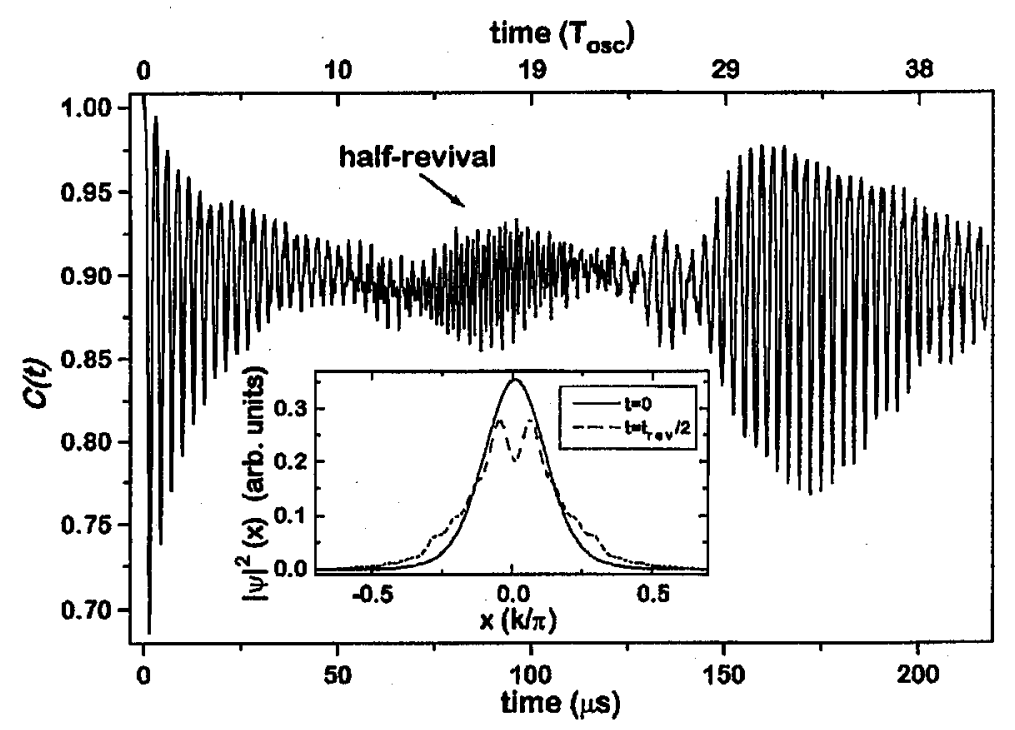

Fig. 5. Model wave packet dynamics showing a clear revival and a clear half-revival. The inset shows the wave packet shape at the half revival point (dashed line) and at the full revival. Note the characteristic double peaked structure at the half-revival signifying the presence of two wave packets which cause the oscillation frequency to be doubled at the half-revival point.

packet and the norm of the wave packet at the peak of the half revival. At the half revival time, a clear double peaked structure is seen, as expected for the spatial wave packet structure at a half revival.

\section{Many pairs of atoms + many photons I: cold collisions}

One very active area of research involves the study of ultra-cold collisions [19]. Experimental and theoretical studies of cold collisions in optical traps have provided a remarkably rich view of what might have appeared to be a simple reaction: the binary interaction between two identical atoms interacting with a laser field.

Many of the interesting properties of ultra-cold collisions which make them different from collisions that take place at high temperature stem from the fact that in the MOT the thermal de Broglie wavelength of the atom $\lambda_{\mathrm{dB}} \approx 5-50 \mathrm{~nm}$. This makes $\lambda_{\mathrm{dB}}$ much larger on the scale of variation of the interatomic collision potentials and far greater than the $s$-wave scattering length, $a_{s} \approx 1-10 \mathrm{~nm}$. This hierarchy defines these vapors as "quantum gases" meaning that the atoms can exhibit highly wave-like behavior in their interactions but are clearly nondegenerate. A separate feature of ultra-cold collisions is that the scale of the interatomic potential energy is large as compared to the average kinetic energy of atoms in the trap, even at larger internuclear separations. Indeed, the collision partners in a MOT measurably experience the long-range van der Waals interactions at interatomic separations of hundreds or even thousands of angströms when dressed by a nearly resonance laser field. In recent years these features of ultra-cold collisions 
have been put to excellent use and researchers have demonstrated the ability to probe long-range atom-atom interactions with exquisite resolution. Perhaps the most important point about ultra-cold collisions is that they occur on a time scale, $\tau_{\text {coll }} \approx 100 \mathrm{~ns} \gg \tau_{\mathrm{sp}} \approx 10 \mathrm{~ns}$. This fact allows for an exciting new possibility: that both absorption and emission processes can modify the collision process. In an ultra-cold collision both the absorbed and emitted photons are able to change the collision, or reaction, pathway during the ongoing collision process. In this way the photons can play a role much more similar to that of a "reaction constituent" rather than the more familiar role as a means of state preparation or interrogation.

One particularly interesting cold collision process that has been investigated by several groups is photoassociative ionization (PAI). Photoassociative ionization is a two-step process in which two colliding atoms absorb two photons sequentially after which the colliding pair ejects an electron through an Auger-like process and produces a bound molecular ion as the final state. In essence, PAI of ultra-cold atonus represents another form of quantum control, namely, the use of laser light to activate and mediate the creation of a molecule starting from the monatomic vapor.

PAI is described schematically in Fig. 6. To date PAI has been primarily studied in ultra-cold $\mathrm{Na}$ collisions and in Fig. $7 \mathrm{a}$ we show a set of interatomic interaction potentials which can be used to describe this collision. In the MOT, the collision begins as two atoms in their ground state approach one another. At

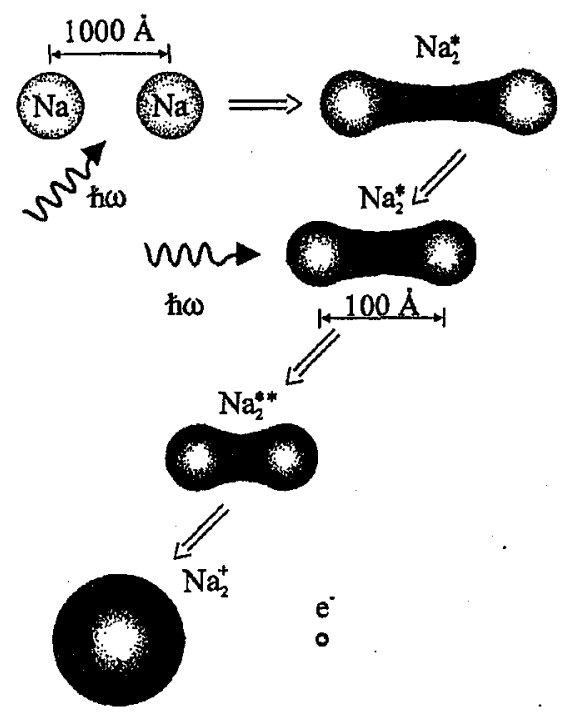

Fig. 6. Photoassociative ionization begins as two atoms approach each other and are excited into a long-range quasi-molecule. This state evolves until the atoms are at a closer range where the molecule can be further excited into a doubly excited state. This doubly excited intermediate state can then autoionize forming a molecular ion. 

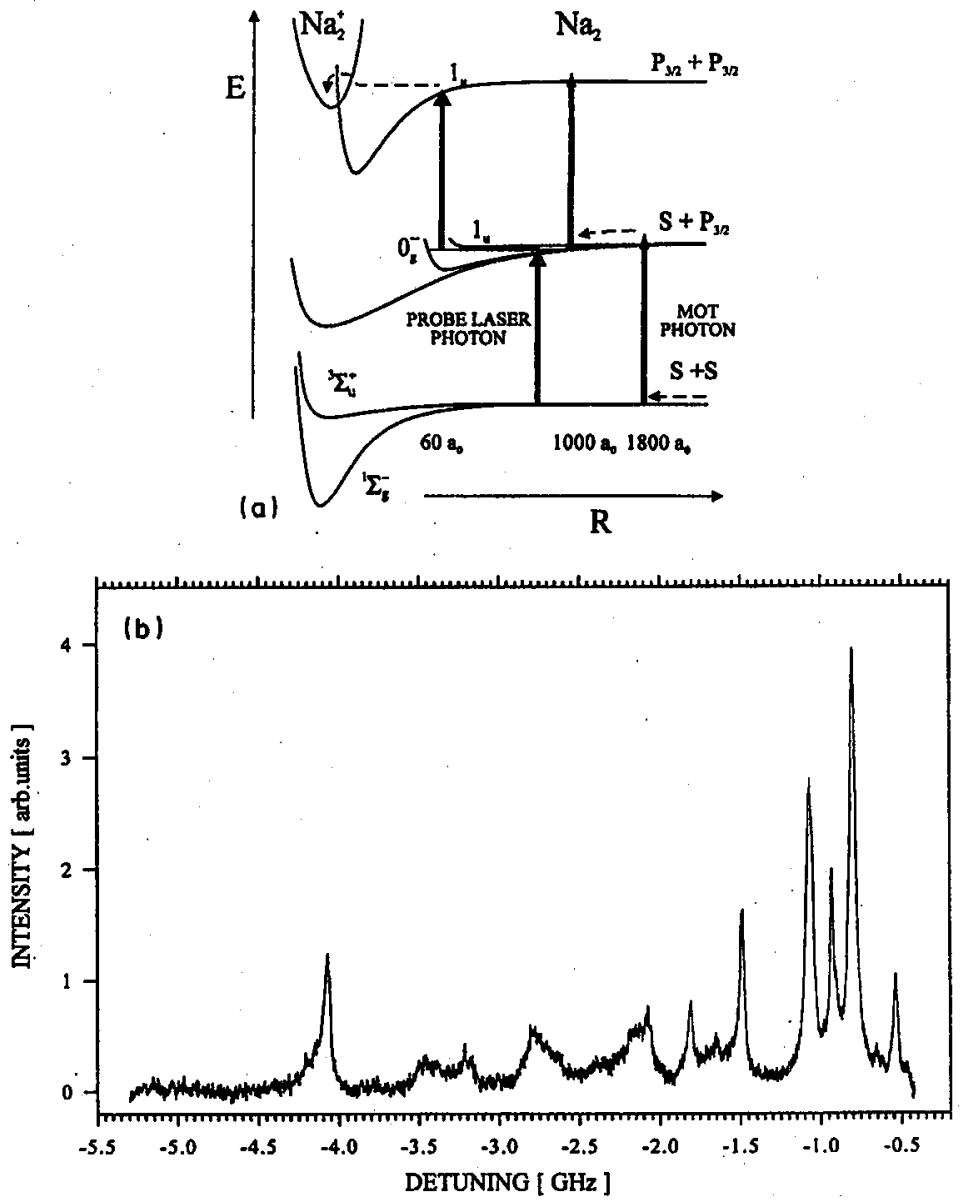

Fig. 7. (a) The intermolecular potential curves and the laser fields describing the photoassociative ionization process illustrated in Fig. 9. $R$ is the internuclear separation. Processes involving trap photons are shown by the two rightmost upward arrows whereas those explored with the second probe laser are the leftmost two upward arrows. This second configuration was used to generate the spectrum shown in Fig. 10b. (b) A portion of the photoassociative ionization spectrum obtained from a $\mathrm{Na}$ trap. The zero of the spectrum is at the $F=2$ to $F^{\prime}=3$ transition on the $D 2$ line of $\mathrm{Na}$. The probe laser intensity for this spectrum is $1 \mathrm{~W} / \mathrm{cm}^{2}$.

remarkably large internuclear spacings $\left(R \approx 1800 a_{0}\right)$ a trap photon is absorbed. The approaching pair then interacts through a very long-range resonant dipole interaction and begins to accelerate toward each other. Some time later, the pair comes back into resonance with the trap lasers and can absorb a second photon $\left(R \approx 1000 a_{0}\right)$. When this happens, the doubly excited pair can then either dissociate or autoionize leading to the final ionic molecule. 
There are a wealth of exciting processes which can be investigated in just this one ultra-cold collision process alone. Let us consider only one of those here: the spectroscopy of the long-range $O_{\mathrm{g}}^{-}$state. Using a second tunable laser as a probe for linear spectroscopy, both the tightly bound state structure of this novel long-range state can be probed as can the near dissociation limit levels and their coupling to the doubly excited $1_{u}$ state. Groups at NIST, Maryland, in Brazil and in Utrecht have carried out elegant spectroscopic measurements of this type as has our group at Rochester. The experimental efforts have been paralleled by extensive theoretical work and a remarkable coherence between experiment and theory has been achieved [20]. The richness of this one type of PAI spectroscopy is characterized in the spectrum shown in Fig. $7 \mathrm{~b}$, taken in our laboratory. This spectrum displays several striking features. For example, the narrowest peak widths are $200 \mu \mathrm{K}$ wide and the spectrum begins $24 \mathrm{mK}$ below the dissociation limit. Furthermore, the structures in this spectrum show clearly that the PAI process involves photoassociation of the atoms into well resolved intermediate molecular states which are very long-range (internuclear spacings of hundreds of Bohr!).

\section{Many pairs of atoms + many photons II: heteronuclear cold collisions}

PAI is only one of many sides of the cold collision problem. Most recently, a group at the University of São Paulo in Brazil and our group at Rochester have opened the door to a new class of ultra-cold collision experiments: we have realized novel multiple species optical traps (see Fig. 8) [21].

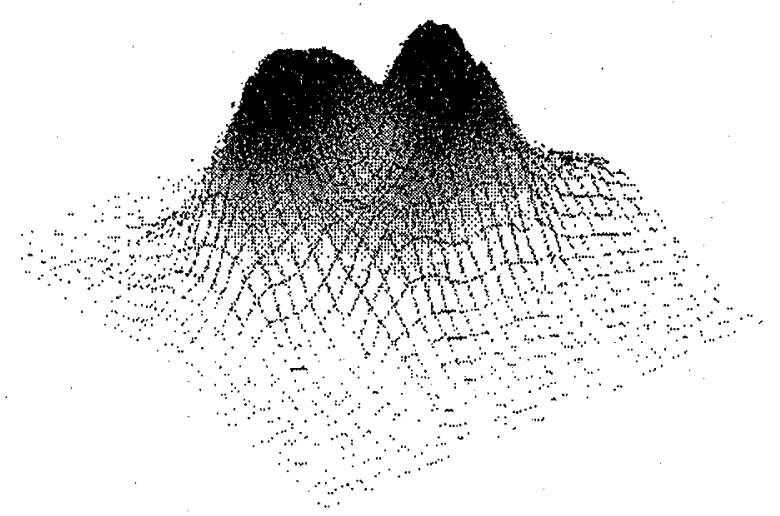

Fig. 8. An image of the trapped atom cloud in a two-species magneto-optical trap. Here the two species are cesium and sodium and the cloud centers have been intentionally displaced.

In our group in Rochester, we have investigated traps composed of different combinations of three alkalis: $\mathrm{Na}, \mathrm{Rb}$ and $\mathrm{Cs}$. Unfortunately, little is known about the interatomic potentials for any of these bi-alkali pairs and hence little is known about collision cross-sections, scattering lengths etc. An important starting point then is to determine the degree of coupling expected between the different 

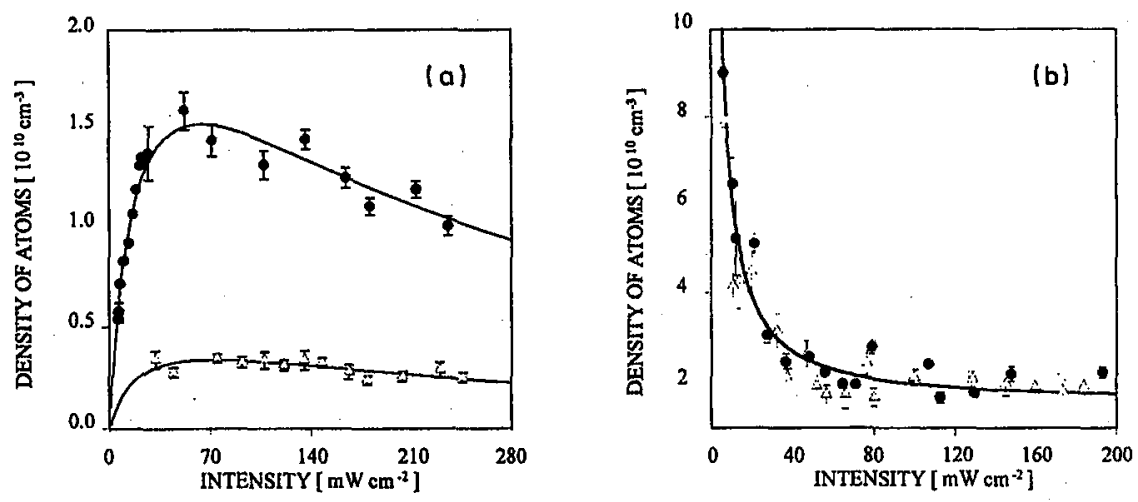

Fig. 9. Intensity dependence of trapped atom number density in two-species $\mathrm{Na}-\mathrm{Cs}$ trap. The left curve is the sodium density as a function of the total intensity of the sodium trapping light while the left curve is the cesium atom density as a function of the total intensity of the cesium trapping light. The solid circles are for the single species traps (i.e. pure $\mathrm{Na}$ on the left and pure $\mathrm{Cs}$ on the right) whereas the triangles are for the two-species trap.

ultra-cold vapors in the trap. In the case of a binary mixture of sodium and cesium atoms, we have found experimentally that the coupling is strong! In Fig. 9a we show a plot of the density of sodium atoms contained within the trap as a function of sodium trap light intensity. The solid circles are for a trap containing pure sodium and the open triangles are for a trap containing a mixture of sodium and cesium atoms. In Fig. 9b we show a similar plot for the number density of trapped cesium atoms. Clearly, although the cesium vapor is almost unaffected by the presence of sodium in the trap, the sodium vapor is greatly affected by the cesium atoms. In fact, at high laser intensities, the density of sodium atoms decreases by almost an order of magnitude when cesium is introduced into the trap. We find experimentally that these effects require two ingredients: (1) that ultra-cold atoms of both species be in the trap and (2) that some of the atoms are in their excited state. We note that we have eliminated the possibility that the sodium vapor density is modified by the cesium trapping light alone.

The measurements described by this data clearly show that there is a strong coupling of the two species in this novel MOT, but they do not provide much information about what the microscopic processes are which cause the loss of sodium. One measurement which does provide more insight, as mentioned above, is photoassociative ionization. Applying the same ion detection techniques used to investigate the pure sodium trap, we have investigated the production of ions in our multi-species traps. For the case of the sodium-cesium mixtures another remarkable change was observed: despite the fact that the trapped sodium atom density is lower in the $\mathrm{Na}-\mathrm{Cs}$ mixture, the flux of ions was observed to increase, and by almost one order of magnitude! Using a time-of-flight technique to mass analyze the ions we find that not only are we observing the production of $\mathrm{Na}_{2}^{+}$ions, but also $\mathrm{Cs}^{+}$ions and, most importantly, $\mathrm{NaCs}^{+}$heteronuclear ions. Simply put, 
using laser cooling and trapping techniques we have demonstrated the ability to "build" heteronuclear molecules! We are currently carrying out detailed spectroscopic measurements to determine the states of the atoms and the molecules which are involved in the heteronuclear PAI process. One particularly exciting possibility is that we can use PAI spectroscopy to determine the two-species $s$-wave scattering length, much as has been done for sodium-sodium and for rubidium-rubidium collisions. These interaction parameters are particularly important for research on the creation and control of another interesting quantum state: the Bose-Einstein condensate.

\section{Many identical atoms: Bose-Einstein condensation}

In the proceedings of the 1993 meeting "Quantum Optics III" a paper by Lewenstein et al. [22] marked the emergence of a new problem in the quantum optics community: that of the Quantum Optics of a Bose-Einstein condensation (BEC) of a dilute, cooled and trapped atomic vapor [23]. In this meeting, several excellent seminars were presented on $\mathrm{BEC}$ and we refer the reader to these papers for some excellent and more detailed discussion.

One of earliest successes of trapped alkali BEC has been in the excellent agreement between the experimental data and the theoretical results derived using a mean-field theory. A representation of the condensate wave function in the mean field picture can be derived from the Gross-Pitaevskii (GP) equation. Essentially, this is a Schrödinger equation for the many-particle ground state (the BEC) with an additional term in the Hamiltonian which is of the form: $U_{0}|\Psi|^{2}$. Here $\Psi$ is the BEC wave function and $U_{0}$ is the interaction potential between the atoms in the condensate. In many situations $U_{0} \propto a_{s}$ where $a_{s}$ is the $s$-wave scattering length of the particles. Frequently it is simplest to solve the GP equation in the so-called Thomas-Fermi approximation which essentially means that the kinetic energy term of the Hamiltonian can be neglected. At Rochester, we have developed a new numerical technique based on a variational approach for solving the GP equation without making any approximations [24]. In most situations, the modifications to the shape of $\Psi$ due to the inclusion of the kinetic energy are small. In fact, only in regions where there is a large curvature in $\Psi$ (and hence a large contribution from the $\nabla^{2} \Psi$ term in the Hamiltonian) does the Thomas-Fermi approximation cause problems. It is not surprising that these problems can become most marked near the condensate boundaries.

\section{Many not completely identical atoms: two-species Bose-Einstein condensation}

With the advent of multi-species traps and the recent realization of a Bose condensate comprised of two different spin-states of a vapor of rubidium atoms [25] a new and important question has arisen: what is the nature of a two-species Bose-Einstein condensate - the $2 B E C$ ? In approaching this question it is important to realize that we cannot expect that each condensate will simply co-exist with the other condensate. The reason for this is that, even though the alkali BECs are very dilute $\left(\approx 10^{13}\right.$ atoms/cc) the role of interparticle interactions is still crucial. For a $2 \mathrm{BEC}$, then, we must consider three classes of interactions: 
the two self-interactions of each species and a third "cross-species" interaction. In the mean field limit, this means that the 2BEC can be characterized by two coupled GP equations and three scattering lengths $a_{11}, a_{22}$ and $a_{12}$ (the cross-species scattering length) [26].
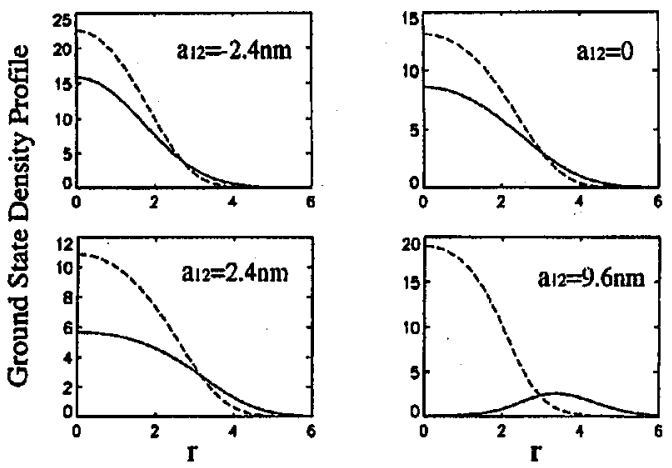

Fig. 10. The ground state density profiles of a two-species Bose-Einstein condensate (2BEC) for two different choices of cross-scattering length $a_{12}$. For this figure we consider a mixture of sodium ( $\mathrm{Na}$ with $a_{11}=3 \mathrm{~nm}$ - the solid lines) and rubidium ( $a_{22}=6 \mathrm{~nm}$ - the dashed lines) atoms. Here $N_{1}=N_{2}=10^{3}$.

A rich array of new phenomena have been predicted for this system including modifications to the ground state wave function profile, the excitation spectrum and even the essential stability of the condensate itself [27]. We have been investigating the $2 \mathrm{BEC}$ using a modification of our variational technique [24]. In Fig. 10 we show the ground state condensate wave function for a mixture of sodium atoms and rubidium atoms contained in a spherically parabolic trap. The parameter which is varied between the different parts of this figure is the cross scattering length $a_{12}$. We see that for strong repulsive interactions (i.e. $a_{12}>0$ ) the ground state is definitely not a mixture of two overlapping condensates, but that instead the system has phase separated into two distinct condensates. Because the ground state condensate wave function in the mean field is the state which minimizes the mean field energy we can provide a physical interpretation of the phase separation. When the system separates, the overlap between the condensates is reduced, and hence the cross-species mean field energy is decreased. Simultaneously, the outer species must be spread over a larger volume, which decreases its mean field energy. By contrast, the mean field energy of the core increases because the core atoms are now closer together (the core atoms are not "diluted" by the second species). Overall, the total system energy is determined by the interplay of these individual energies and in the phase separated state the total mean field energy is minimized. A careful analysis of the JILA two-spin state condensates has provided some evidence for the effective "condensate repulsion", however a true two-species condensate has yet to be realized. At Rochester, an experimental effort on this problem is well underway. 


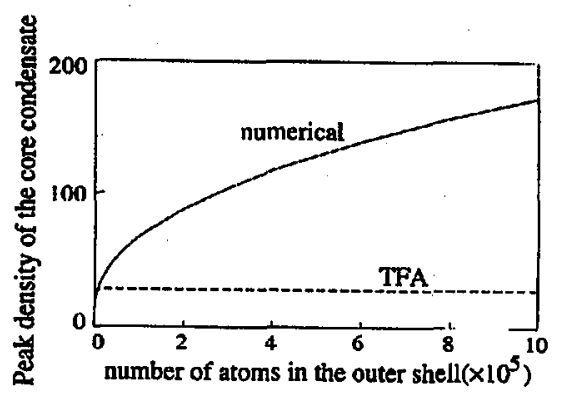

Fig. 11. The density of atoms in the core condensate as a function of the number of atoms in the outer shell condensate. Note that as the number of atoms in the shell increases, the inner condensate is compressed. The dotted line is the density of the core as calculated within the Thomas-Fermi approximation.

In Fig. 11, we show a plot of the density of the core species as a function of the number of atoms in the shell condensate. What is predicted is that as the outer condensate particle number increases, the shell will compress the core condensate. In addition to the prediction of this new phenomenon, this result highlights another point: in the 2BEC, once phase separation has occurred and boundaries have appeared, the Thomas-Fermi approximation does not give reliable results. In the figure, the dotted line is the density as calculated using the Thomas-Fermi approximation and the solid line is for the full GP equation.

\section{Conclusion}

Physicists from many backgrounds have become excited by the problems that laser cooling has brought into view and generations of students are emerging with Ph.D.s earned in the field. Laser coolers have refined the techniques for manipulating individual atoms, and ensembles of atom, the collisions between atoms, and in creating novel macroscopic quantum states. Overall the efforts in quantum optics to control and manipulate the external coordinates of atoms have developed into a remarkable and dynamic field and we are pleased to be part of this excitement.

The list of remarkable scientists that have contributed to our understanding is too large to list - we thank them nevertheless - and we apologize for the many omissions we have made. N.P.B. is particularly grateful to K. Rzązewski for insightful discussions and inspiration early on in our lattice wave packet work. We are grateful to the National Science Foundation, the David and Lucile Packard Foundation and the Laboratory for Laser Energetics for Financial Support. R.E. acknowledges support of the CNPq.

\section{References}

[1] D. Wineland, H. Demelt, Bull. Am. Phys. Soc. 20, 637 (1975); T. Hansch, A. Schalow, Opt. Commun. 13, 68 (1975).

[2] D.J. Wineland, R. Drullinger, F. Walls, Phys. Rev. Lett. 40, 1639 (1978). 
[3] S.V. Andreev, V.I. Balykin, V.S. Letokhov, JETP Lett. 34, 442 (1978).

[4] W.D. Phillips, J.V. Prodan, in: Coherence and Quantum Optics V, Eds. L. Mandel, E. Wolf, Plenum, New York 1984, p. 15.

[5] E.L. Raab, M. Prentiss, A. Cable, S. Chu, D.E. Pritchard, Phys. Rev. Lett. 59, 2631 (1987).

[6] C. Cohen-Tannoudji, in: Proc. Int. School of Physics, Enrico Fermi, course CXVIII, Varenna (Italy) 1991, Eds. E. Arimondo, W.D. Phillips, F. Strumia, North-Holland, New York 1992, p. 99.

[7] J. Dalibard, C. Cohen-Tannoudji, J. Opt. Soc. Am. B 6, 2023 (1989); P. Unger, D. Weiss, E. Riis, S. Chu, J. Opt. Soc. Am. B 6, 2058 (1989); J. Dalibard, C. Salomon, A. Aspect, E. Arimondo, R. Kaiser, N. Vansteenkiste, C. Cohen-Tannoudji, in: Atomic Physics, Vol. 11, Eds. S. Haroche, J.C. Gray, G. Grynberg, World Scientific, Singapore 1989, p. 199.

[8] C.J. Myatt, N.R. Newbury, R.W. Ghrist, S. Loutzenhiser, C.E. Wieman, Opt. Lett. 21, 290 (1996).

[9] G. Brikl, M. Gatzke, I.H. Deutsch, S. Rolston, W.D. Phillips, Phys. Rev. Lett. 75, 2823 (1995); M. Weidemuller, A. Hemmerich, A. Gorlitz, T. Esslinger, T.W. Hansch, Phys. Rev. Lett. 75, 4583 (1995).

[10] C.I. Westbrook, R.N. Watts, C.E. Tanner, S.L. Rolston, W.D. Phillips, P.D. Lett, P.L. Gould, Phys. Rev. Lett. 65, 33 (1990); N.P. Bigelow, M. Prentiss, Phys. Rev. Lett. 65, 29 (1990); P.S. Jessen, C. Gerz, P.D. Lett, W.D. Phillips, S.L. Rolston, R.J.C. Spreeuw, C.I. Westbrook, Phys. Rev. Lett. 69, 49 (1992).

[11] R. Dicke, Phys. Rev. 89, 472 (1953).

[12] K.I. Petsas, A.B. Coates, G. Grynberg, Phys. Rev. A 50, 5173 (1994).

[13] M. Raizen, C. Salomon, Q. Niu, Phys. Today 50, 30 (1997).

[14] M.B. Dahan, E. Peik, J. Reichel, Y. Castin, C. Salomon, Phys. Rev. Lett. 76, 4508 (1996).

[15] Q. Niu, X.-G. Zhao, G.A. Geogakis, M.G. Raizen, Phys. Rev. Lett. 76, 4504 (1996); S.R. Wilkinson, C.F. Bharucha, K.W. Madison, Q. Niu, M.G. Raizen, Phys. Rev. Lett. 76, 4512 (1996).

[16] M. Kozuma, N. Nakagawa, W. Jhe, M. Ohtsu, Phys. Rev. Lett. 76, 2428 (1996); G. Raithel, G. Birkl, W.D. Phillips, S.L. Rolston, Phys. Rev. Lett. 78, 2928 (1997); A. Gorlitz, W. Weidemuller, T.W. Hansch, A. Hemmerich, Phys. Rev. Lett. 78, 2096 (1997); P. Rudy, R. Ejnisma, N.P. Bigelow, Phys. Rev. Lett. 78, 4906 (1997).

[17] J.H. Eberly, N.B. Narozhny, J.J. Sanchez-Mondragon, Phys. Rev. Lett. 44, 1323 (1980).

[18] R. Ejnisman, P. Rudy, H. Pu, N.P. Bigelow, Phys. Rev. A 56, 4331 (1997).

[19] See for example, P. Julienne, in Ref. [6], p. 733; T. Walker, Adv. Atom. Mol. Opt. Phys. 34, 125 (1994); P.S. Julienne, J. Natl. Inst. Stand. Technol. 101, 487 (1996). 
[20] Recent experiments include those by P.D. Lett, K. Helmerson, W.D. Phillips, L.P. Ratcliffe, S.L. Rolston, M.E. Wagshul, Phys. Rev. Lett. 71, 2200 (1993); V. Bagnato, L. Marcassa, C. Tsao, Y. Wang, J. Weiner, Phys. Rev. Lett. 70, 3225 (1993); J.J. Blange, J.M. Zijlstra, A. Amelink, X. Urbain, H. Rudolph, P. van der Straten, H.C. Beijerinck, H.G.M. Hiedeman, Phys. Rev. Lett. 78, 3089 (1997). Many aspects of the theoretical work are summarized in R.W. Heather, P.S. Julienne, Phys. Rev. A 47, 1887 (1993) and references therein.

[21] M.S. Santos, P. Nussenzweig, L.G. Marcassa, K. Helmerson, J. Flemming, S.C. Zilio, V.S. Bagnato, Phys. Rev. A 52, 4340 (1995); J.P. Shaffer, N.P. Bigelow, Opt. Photon. News 12, 46 (1995).

[22] M. Lewenstein, L. You, J. Cooper, Acta Phys. Pol. A 86, 173 (1994).

[23] M.H. Anderson, J.R. Ensher, M.R. Mathews, C.E. Wieman, E.A. Cornell, Science 269, 198 (1995); C.C. Bradley, C.A. Sackett, J.J. Tollett, R.G. Hulet, Phys. Rev. Lett. 75, 1687 (1995); K.B. Davis, M.-O. Mews, M.R. Andrews, N.J. van Druten, D.S. Durfee, D.M. Kurn, W. Ketterle, Phys. Rev. Lett. 75, 3969 (1995).

[24] H. Pu, N.P. Bigelow, submitted to Phys. Rev. Lett.

[25] C.J. Myatt, E.A. Burt, R.W. Ghrist, E.A. Cornell, C.E. Weiman, Phys. Rev. Lett. 78, 586 (1997).

[26] Tin-Lun Ho, V.B. Shenoy, Phys. Rev. Lett. 77, 3276 (1996); see also B.D. Esry, C.H. Green, J.P. Burke, J.L. Bohn, Phys. Rev. Lett. 78, 3594 (1997) and E.V. Goldstein, P. Meystre, Phys. Rev. A 55, 2935 (1997).

[27] C.K. Law, H. Pu, N.P. Bigelow, J.H. Eberly, Phys. Rev. Lett. 79, 3105 (1997). 